\title{
Experimental and Simulation Study of the Optical Performances of a Wide Grid Polarizer as a Luminance Enhancement Film for LCD Backlight Applications
}

\author{
Jae Seok Seo, Tae Eun Yeom, and Jae-Hyeon Ko* \\ Department of Physics, Hallym University, Chuncheon 200-702, Korea
}

(Received April 9, 2012 : accepted May 14, 2012)

\begin{abstract}
Reflective polarizers can be used as luminance enhancement films for LCD backlights via the polarization recycling process. The optical performances of a wire grid polarizer (WGP) as a reflective polarizer adopted in edge-lit backlights were investigated by luminance evaluation and a time-domain simulation technique. The results were compared to those of a commercial dielectric multilayer film. The luminance gain factor of WGP was smaller than that of the multilayer film by $18 \%$. This was attributed to a much larger internal loss of WGP due to light absorption by metal wires. The internal losses of both reflective polarizers and the polarization conversion efficiency of the backlight were obtained numerically based on a phenomenological model. The optical performances of WGP were optimized by using a time-domain simulation technique. The luminance gain increased and was found to become comparable to, but slightly less than the case of the dielectric multilayer film with decreasing line width.
\end{abstract}

Keywords : Backlight unit (BLU), Simulation, Luminance enhancement, Wire grid polarizer, Liquid crystal display (LCD)

OCIS codes : (120.2040) Displays; (150.2950) Illumination; (230.3720) Liquid-crystal devices

\section{INTRODUCTION}

The LCD (Liquid Crystal Display) is the most typical example of non-emissive displays requiring an independent light source called BLU (Backlight Unit). BLU supplies LCD with homogeneous, bright, white light with appropriate color characteristics. Light sources in BLU generate visible light while other optical components diffuse, homogenize and collimate the generated light [1]. The importance of BLU technology has been increased recently because of its key role in the innovation of LCD, in particular, its largesize applications. Recent large-size LCD TV's adopt not only direct-lit but also edge-lit LED(light emitting diode) backlights the latter making the TV much slimmer and lighter along with reducing power consumption [2-3]. Moreover, dynamic driving of BLU may be adopted to improve the picture quality of LCD substantially.

The transmission of LCD is usually less than $10 \%$, which means the luminance of BLU should be at least 10 times higher than the panel luminance. The power consumption of TVs should be minimized to satisfy energy regulation policy. This requires that highly-efficient optical films be used to maximize the utilization of visible light generated from light sources. The most representative brightness enhancement films are collimation films and reflective polarizers (RPs). Collimation films such as prism films and micro-lens films refract the incident light toward the normal direction resulting in the increase in the on-axis luminance at the expanse of high-angle luminance values $[4,5]$. On the other hand, RPs reflect the light having the polarization component orthogonal to that parallel to the transmission axis of the bottom polarizer of LCD [5]. Figure 1 is a schematic diagram that shows the operating principle of RPs in backlights. Let's assume that the polarization component denoted as $I_{\mathrm{H}}$ passes through the bottom polarizer of LCD while the orthogonal $I_{\mathrm{V}}$ component is absorbed. If a reflective polarizer is inserted between the backlight and the bottom polarizer of LCD, it reflects the $I_{\mathrm{V}}$ component downward to the backlight. The polarization state of the reflected light $\left(I_{\mathrm{V}}\right)$ is partly transformed into the $I_{\mathrm{H}}$ component that contributes to the enhancement of the luminance on the LCD panel.

\footnotetext{
*Corresponding author: hwangko@hallym.ac.kr

Color versions of one or more of the figures in this paper are available online.
} 


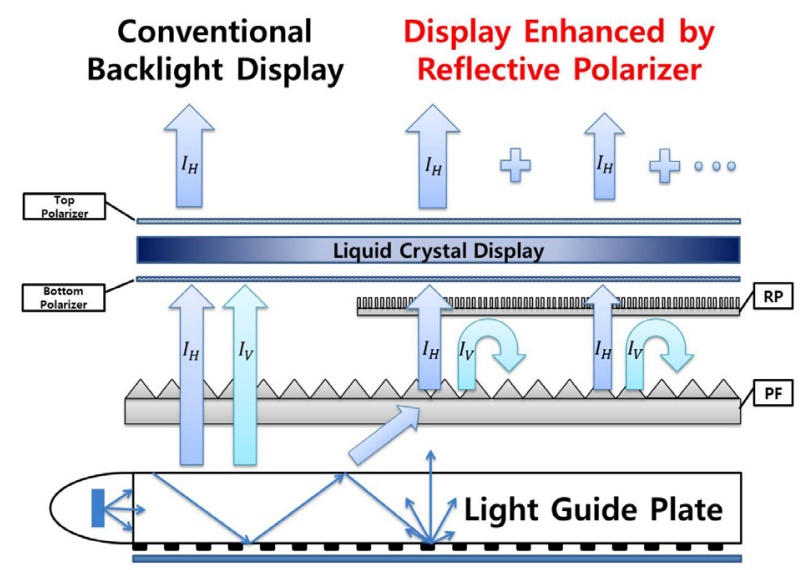

FIG. 1. A schematic figure depicting the operation principle of the reflective polarizer in LCD backlights. RP and PF denote the reflective polarizer and the prism film, respectively.

Several optical principles are used to make efficient RPs. Among them, birefringent multilayer films based on generalized Fresnel relations [6] have been developed and adopted in BLU. Anisotropic scattering films [7], wire grid-based polarizers (WGPs) [8], and cholesteric liquid crystal (CLC) films [9] have been studied and developed to replace the costly multi-layer films. The optical performances of the commercially-available birefringent multilayer film in several kinds of BLU have been reported in detail [10-13]. However, the optical performances of new RPs such as WGP and CLC films have not been studied in detail as a brightness enhancement films in backlights. The current study is to report the optical performances of WGP as a RP in the edge-lit BLU for the first time and to compare them with those of commercial dielectric multilayer films. The FDTD (finite difference time domain) simulation method was used to optimize and predict the optical performance of WGPs as a RP in BLU.

\section{EXPERIMENT AND SIMULATION}

A 3.5-inch edge-lit BLU consisting of white LEDs, a light guide plate (LGP), a diffuser film (DF), and a prism film(PF) was used to evaluate the optical performances of a WGP. A spectroradiometer (PR670, Photo Research) was used to measure the luminance. A simple home-made rotator was used to obtain the angular dependence of the luminance on each film. In order to mimic the bottom polarizer of LCD, an absorptive sheet polarizer (AP) was put on the PF. Either a multi-layer RP (DBEF-D, 3M Co.) or a WGP (NT46-636, Edmund Optics) was inserted between PF and AP. The pitch, height, and the width of wire grids on the WGP were $144 \mathrm{~nm}, 155 \mathrm{~nm}$, and $65 \mathrm{~nm}$, respectively. The on-axis luminance was measured before and after inserting the RP to determine the luminance gain factor due to the polarization recycling process. On the other hand, the con-

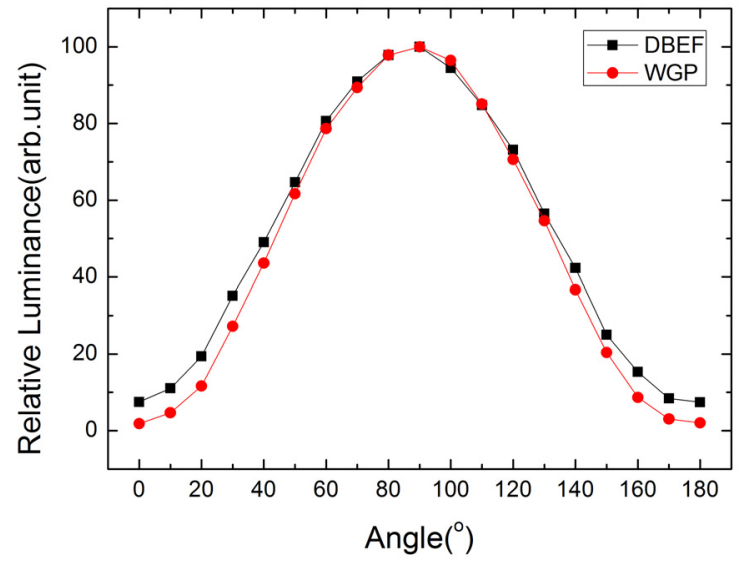

FIG. 2. The angular dependence of the normalized luminance on the rotation angle of the absorptive polarizer (AP) with respective to the fixed reflective polarizer (RP). $90^{\circ}$ indicates the condition that the transmission axis of the RP is parallel to that of the AP.

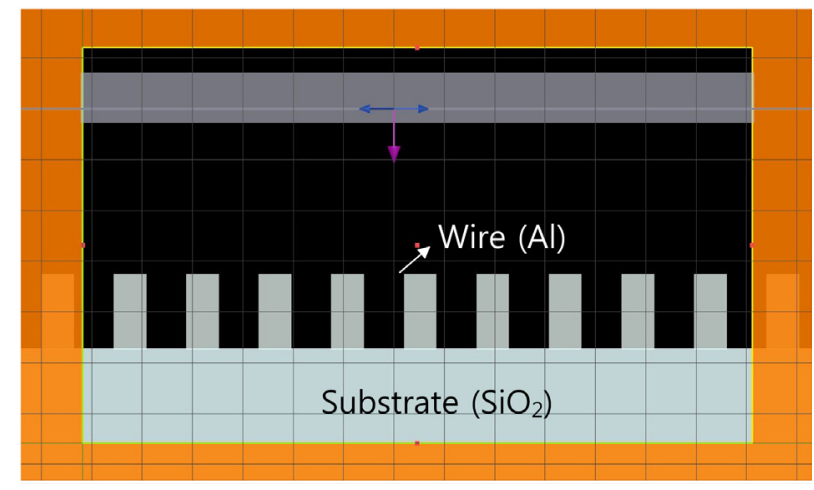

FIG. 3. One example of the two-dimensional WGP with a width of $65 \mathrm{~nm}$, a height of $155 \mathrm{~nm}$ and a period of $144 \mathrm{~nm}$ constructed by the FDTD Solution software.

trast ratio of each RP was obtained by rotating the transmission axis of the AP put over the RP. Figure 2 shows the angular dependence of the luminance on the rotation angle of the AP. $90^{\circ}$ indicates the condition that the transmission axis of the RP is parallel to that of the AP. The contrast ratios of DBEF and WGP were about 13 and 52, respectively.

The FDTD Solution software (Ver.7.5, Lumerical Co.) was used to simulate the optical performances of WGP. This method is a time domain technique by which the electric and magnetic fields can be calculated as a function of time based on the Maxwell equations. The frequency information is obtained via Fourier transformation during the simulation. The simulation was carried out in a twodimensional space. The pitch, the width and the height of the grids were changed systematically to investigate the dependence of the optical performances such as transmission on the geometrical parameters of WGP. Figure 3 shows one example of the constructed WGP with a width 
of $65 \mathrm{~nm}$, a height of $155 \mathrm{~nm}$ and a period of $144 \mathrm{~nm}$, which are the dimensions of metal wires formed in the experimental WGP. The materials for the metal wire and the substrate were aluminum and $\mathrm{SiO}_{2}$ glass, respectively. A plane wave at a specific wavelength and a specific polarization state in the visible range was used to investigate its transmission and reflection behaviors depending on the polarization.

\section{RESULTS AND DISCUSSION}

Figures 4 (a) and (b) show the luminance distributions on $\mathrm{LGP}, \mathrm{LGP}+\mathrm{DF}, \mathrm{LGP}+\mathrm{DF}+\mathrm{PF}$, and $\mathrm{LGP}+\mathrm{DF}+\mathrm{PF}+\mathrm{RF}$ (DBEF) along the horizontal and vertical directions, respectively. The experimental results in this figure are very typical in edge-lit BLUs. The light tends to escape toward high-angle directions on LGP due to the specular reflection component of the scattering dots printed on the bottom surface of LGP. These oblique rays are redirected toward the normal direction via DF and PF. Figure 5 shows three angular distributions of the luminance on $\mathrm{AP}, \mathrm{AP}+\mathrm{DBEF}$ and AP+WGP, all of which were installed on $\mathrm{LGP}+\mathrm{DF}$ $+\mathrm{PF}$. The luminance value on AP can be considered to be proportional to the amount of the light that passes through

(a)

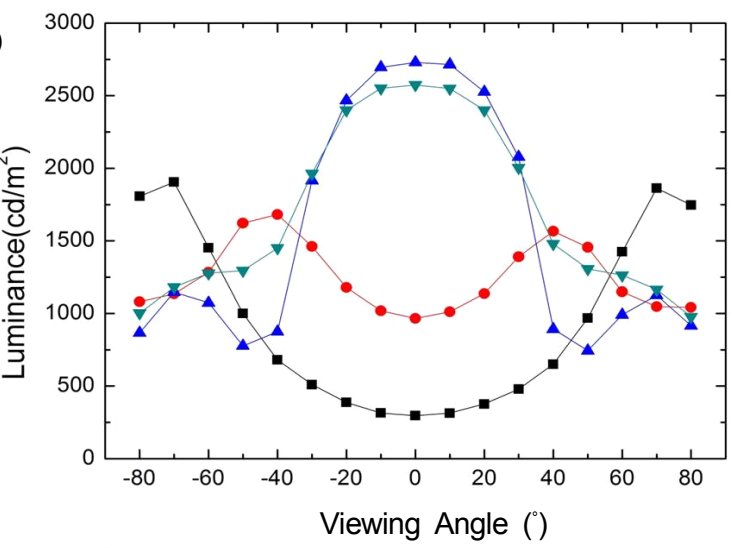

(b)

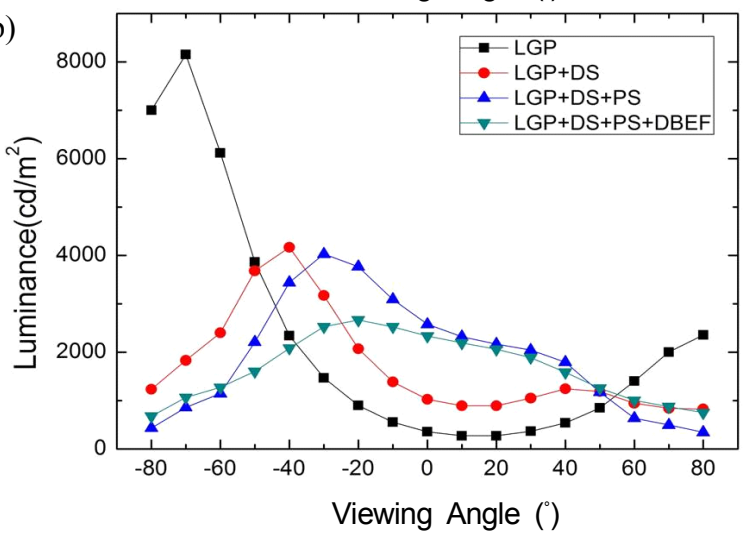

FIG. 4. The angular luminance distributions on LGP, $\mathrm{LGP}+\mathrm{DF}, \mathrm{LGP}+\mathrm{DF}+\mathrm{PF}$, and $\mathrm{LGP}+\mathrm{DF}+\mathrm{PF}+\mathrm{RF}(\mathrm{DBEF})$ along (a) the horizontal and (b) the vertical directions, respectively. the bottom polarizer of the LCD panel. This corresponds to the $I_{\mathrm{H}}$ polarization component in Fig. 1. The other component $\left(I_{\mathrm{V}}\right)$ is mostly absorbed by AP. If DBEF or WGP is inserted between PF and AP, the polarization recycling process for the $I_{\mathrm{V}}$ component occurs, and the luminance value is expected to increase. Fig. 5 clearly shows that the luminance on the LCD panel has been increased substantially by adopting a RP and the resultant polarization recycling effect. However, the luminance gain factor of DBEF looks larger than that of WGP in spite of the higher contrast ratio of WGP. The numerical values of the luminance gain factor are shown in Table 1.

A phenomenological model [5] can be used to evaluate the detailed performances of RP [11-13]. Figure 6 shows a schematic figure of this model. According to this model, the incident, unpolarized light $\left(I_{\mathrm{H}}+I_{\mathrm{V}}\right)$ from BLU onto RP is partly transmitted (with a transmittance of $t$ ) and partly reflected (with a reflectance of $r$ ) depending on the polarization state. $I_{\mathrm{H}}$ and $I_{\mathrm{V}}$ may here be considered as optical power or luminous flux (thus proportional to the luminance) of the transmitted and reflected polarization components, respectively, at RP. If the loss factor in RF is denoted as

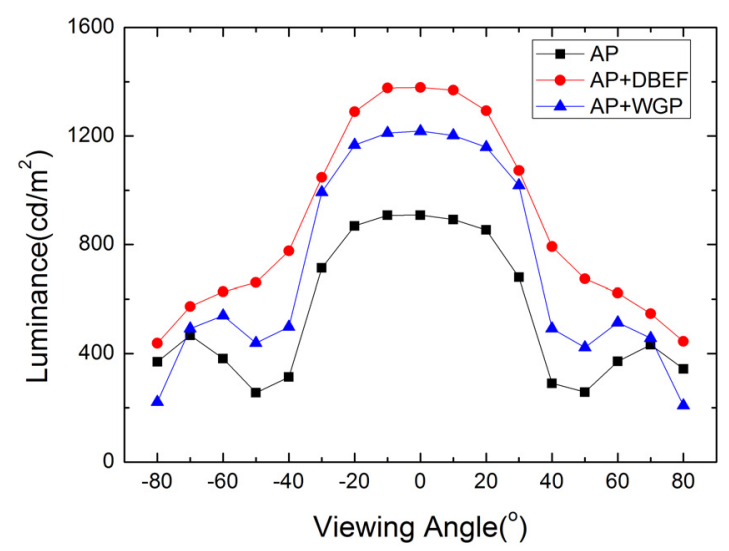

FIG. 5. Angular distributions of the luminance on AP, $\mathrm{AP}+\mathrm{DBEF}$ and AP+WGP, each was put on the edge-lit backlight consisting of $\mathrm{LGP}+\mathrm{DF}+\mathrm{PF}$.

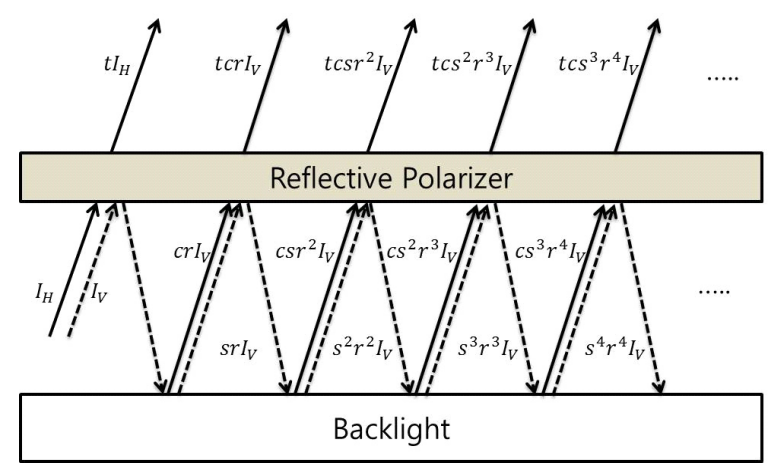

FIG. 6. A schematic figure showing the operating principle of reflective polarizers [5]. Regarding the meaning of symbols, see the text. 
TABLE 1. Luminance values on an absorptive polarizer that was placed on two types of reflective polarizers(RPs), i.e., DBEF or WGP, in an edge-lit backlight. The transmission axis of the absorptive polarizer was set to be parallel to that of RP. The gain factor $G$, the conversion ratio $c$, and the loss factor $L_{\mathrm{c}}$ and $L_{\mathrm{RP}}$ of the backlight and RP, respectively, are also shown in the table

\begin{tabular}{c|c|c|c|c|c|c}
\hline \hline $\begin{array}{c}\text { Combination of } \\
\text { optical films }\end{array}$ & $\begin{array}{c}\text { Luminance } \\
\text { without RP } \\
\left(\mathrm{cd} / \mathrm{m}^{2}\right)\end{array}$ & $\begin{array}{c}\text { Luminance } \\
\text { with RP } \\
\left(\mathrm{cd} / \mathrm{m}^{2}\right)\end{array}$ & $\begin{array}{c}\text { Luminance } \\
\text { Gain } G\end{array}$ & $\begin{array}{c}\text { Conversion } \\
\text { Ratio } c\end{array}$ & $\begin{array}{c}\text { Loss Factor } \\
(\mathrm{BLU})\end{array}$ & $\begin{array}{c}\text { Loss Factor } \\
(\mathrm{RP}) L_{\mathrm{RP}}\end{array}$ \\
\hline $\begin{array}{c}\text { LGP+DF+PF+AP } \\
(+\mathrm{DBEF})\end{array}$ & 908 & 1379 & 1.52 & $0.38^{\mathrm{a})}$ & $0.24^{\mathrm{a})}$ & 0.03 \\
\hline $\begin{array}{c}\text { LGP+DF+DF+AP } \\
(+\mathrm{WGP})\end{array}$ & 908 & 1218 & 1.34 & & $0.10^{\mathrm{a})}$ \\
\hline
\end{tabular}

a) obtained from FDTD simulation combined with the luminance gain due to WGP.

$L_{\mathrm{RP}}$ for each polarization component, $t+L_{\mathrm{RP}}=1$ and $r+L_{\mathrm{RP}}=1$ according to the energy conservation. The reflected light $\left(I_{\mathrm{V}}\right)$ toward the backlight is partially converted to the $I_{\mathrm{H}}$ component, of which the conversion ratio is denoted as $c$, due to scattering and diffuse reflection, while part of it remains in the $I_{\mathrm{V}}$ polarization state with a probability of $s$. If the loss in the backlight is denoted as $L_{\mathrm{c}}, c+s+L_{\mathrm{c}}=1$. Fig. 6 shows that this conversion process (or polarization recycling process) is repeated infinitely, and the total transmitted power can be expressed by the following equation [5].

$$
\begin{aligned}
P_{\text {total }} & =t I_{H}+t c I_{V} r+t c I_{V} s r^{2}+t c I_{V} s^{2} r^{3}+t c I_{V} s^{3} r^{4}+\cdots \\
& =t I_{H}+t c I_{V} r\left[1+s r+(s r)^{2}+(s r)^{3}+\cdots\right] \\
& =t I_{H}\left[1+\frac{r c\left(I_{V} / I_{H}\right)}{1-s r}\right]
\end{aligned}
$$

According to this equation, the power gain (and thus luminance gain) factor $G\left(P_{\text {total }}\right.$ divided by $\left.I_{\mathrm{H}}\right)$ is given by Eq. (2) [5].

$$
G=t\left(1+\frac{r c\left(I_{V} / I_{H}\right)}{1-s r}\right)
$$

The ratio $I_{\mathrm{V}} / I_{\mathrm{H}}$ was experimentally determined to be 0.95 on PF. For the case of DBEF, it was previously assumed that $t \sim r \sim 1$ with $L_{\mathrm{RP}} \sim 0$ [5]. In this case, the gain factor $G$ is given by $1+c\left(I_{\mathrm{V}} / I_{\mathrm{H}}\right) /(1-s)$. If it is assumed that $c$ is equal to $s$, the numerical values of $G, c$ and $L_{\mathrm{c}}$ can be obtained from experimental results of $G$. Since the luminance gain by DBEF was $1.52, c$ and $L_{\mathrm{c}}$ of the backlight which played the role of the polarization conversion element in this model were 0.35 and 0.29 , respectively. This approach was used in previous studies where the optical performances of DBEF were evaluated [11-13]. However, there is no clear experimental evidence that justifies the assumption of $L_{\mathrm{RP}} \sim$.

In order to determine the value of $L_{\mathrm{RP}}$ for DBEF, $c$ and $L_{\mathrm{c}}$ of the backlight should be determined in advance. Since the same edge-lit backlight was used for two types of reflective polarizer, $c$ and $L_{\mathrm{c}}$ should be the same in both cases. Therefore, the FDTD technique was used to simulate the optical performances of WGP from which $c$ and $L_{\mathrm{c}}$ could be derived. The dimensions of the WGP used in the experiment were adopted to construct a two-dimensional WGP by using the FDTD Solution software. A plane wave at the wavelength of $550 \mathrm{~nm}$ was incident toward the WGP at normal direction. The results for both $I_{\mathrm{H}}$ and $I_{\mathrm{V}}$ polarization components were averaged and showed that $t=r=0.90$ with $L_{\mathrm{RP}}=0.10$ for WGP. This indicates that approximately $10 \%$ of the incident light is lost in WGP due to dissipation. If these values are input in Eq. (2) combined with $G=1.34$, it turns out that the conversion factor $c(=s)=0.38$ and $L_{\mathrm{c}}=0.24$. Since these values should be the same for the case of DBEF-based backlight, $L_{\mathrm{RP}}$ and $t(=r)$ are found to be 0.03 and 0.97 , respectively, for DBEF when the Eq. (2) is used. The comparison of these parameters between DBEF and WGP is shown in Table 1. These results clearly show that the inferior optical performance of WGP as a reflective polarizer in backlights is related to the higher loss and lower transmission of WGP, which is presumably due to the absorption of light in metal wires.

The most important parameters that determine the optical performance of WGP are the period(=pitch), height, and duty cycle(the width divided by the pitch) of metal wires. Nanoimprint lithography is usually used to fabricate nanoWGPs for the purpose of polarization manipulation [8]. Figures 7 (a), (b) and (c) are the dependences of the transmission of $I_{\mathrm{H}}$ polarization on the period, height and duty cycle, respectively, at three wavelengths in the visible range. Fig. 7(a) shows that the transmission of $I_{\mathrm{H}}$ polarization component is more than 0.7 when the period is smaller than $200 \mathrm{~nm}$ at a fixed duty ratio of 0.45 and a height of $155 \mathrm{~nm}$. Although the transmission increases with decreasing period, the minimum period will be limited by the current nanoimprint lithography technology. The $I_{\mathrm{H}}$ transmission at a fixed period of $144 \mathrm{~nm}$ and a height of 155 $\mathrm{nm}$ shown in Fig. 7(b) displays a maximum at each wavelength as a function of the height. The experimental value of the height of WGP (i.e., $144 \mathrm{~nm}$ ) seems a good choice because the $I_{\mathrm{H}}$ transmission values are higher than 0.77 for all three wavelengths. Fig. 7(c) shows that the $I_{\mathrm{H}}$ transmission increases with decreasing duty cycle at a fixed period of 

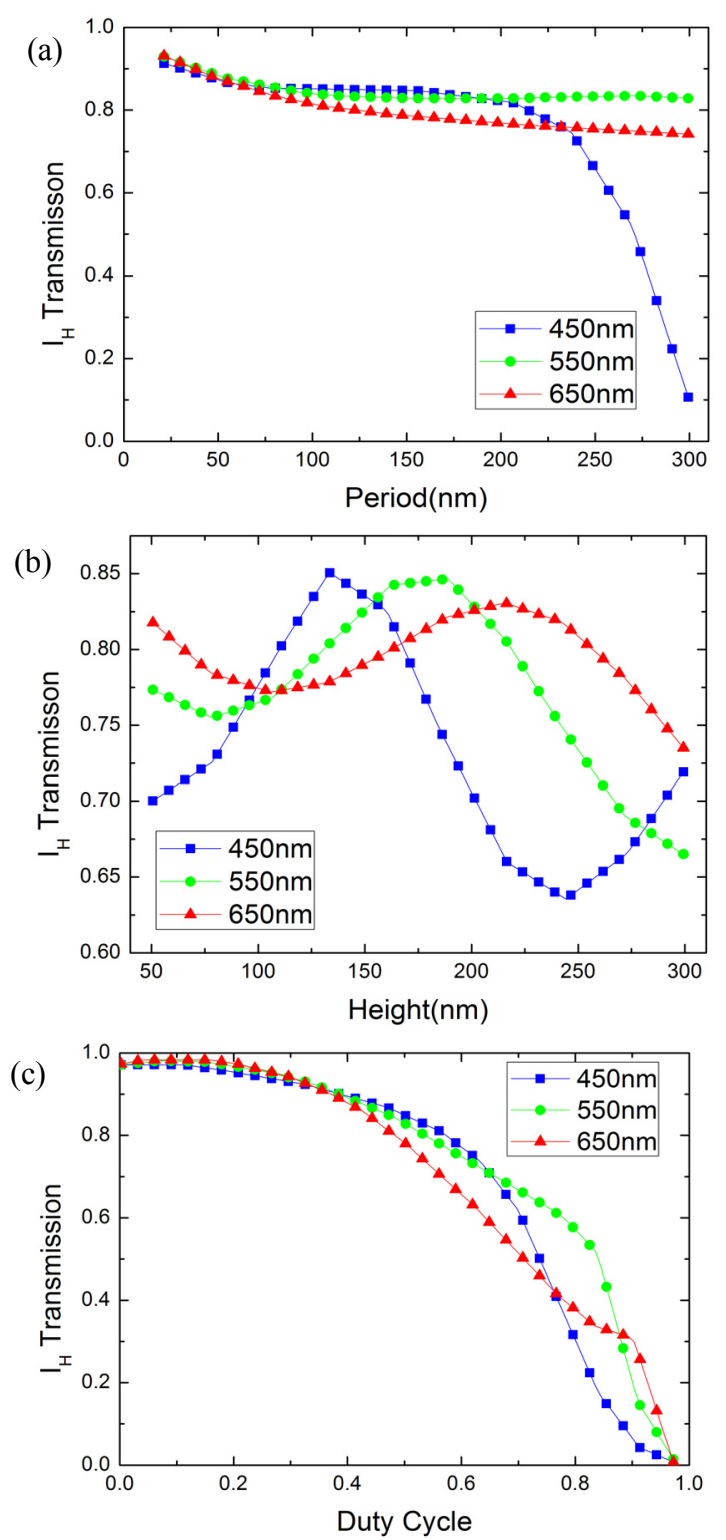

FIG. 7. The dependence of the transmission of $I_{\mathrm{H}}$ polarization on the period, height and duty ratio, respectively.

$144 \mathrm{~nm}$, indicating energy efficiency will be increased with smaller width if the period is fixed. The duty cycle of 0.5 means that the wire width is $72 \mathrm{~nm}$, i.e., half the period of $144 \mathrm{~nm}$. The minimum allowed duty cycle will also be limited by the manufacturing process. These simulation results are consistent with previous studies $[8,14]$.

Based on these simulation results, the best performance of WGP as a reflective polarizer in the backlight can be discussed. For this purpose, the height was fixed to the experimental value of $155 \mathrm{~nm}$, and the width (i.e., duty cycle) or the period was changed to investigate the dependence of the luminance gain $G$ on these parameters. Figures 8 (a) and (b) are the simulation results for the gain factor as functions of the duty cycle and the period, respectively, obtained for the plane waves at the wave-
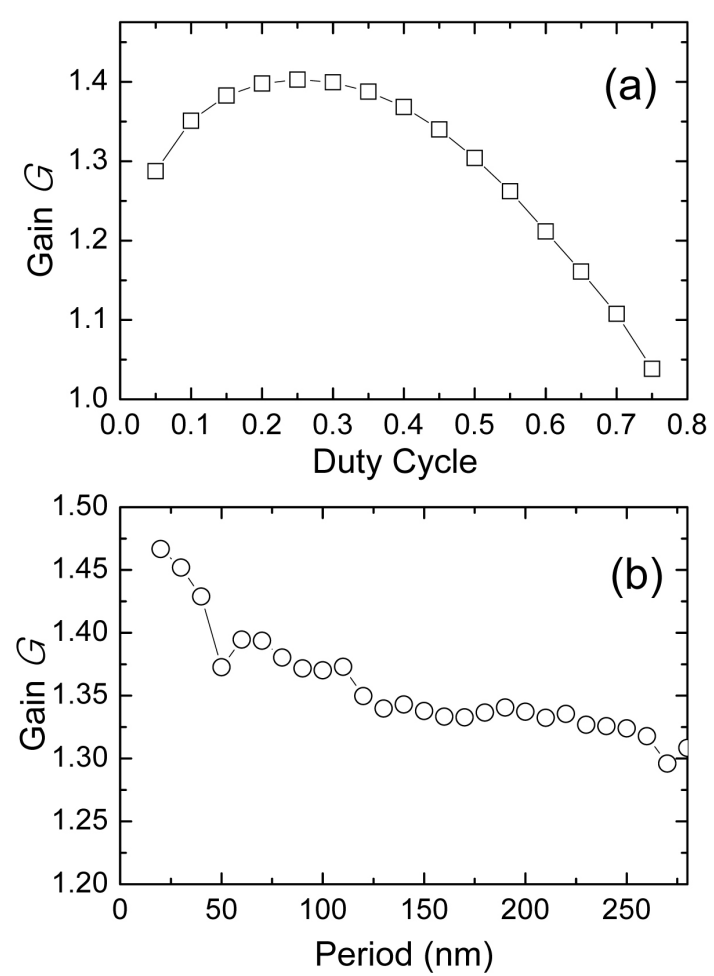

FIG. 8. The simulation results for the gain factor as functions of (a) the duty cycle and (b) the period obtained for the plane waves at the wavelength of $550 \mathrm{~nm}$.

length of $550 \mathrm{~nm}$. The optimum duty ratio for the maximum gain is 0.25 at which the luminance gain is about 1.4 and the wire width is $\sim 36 \mathrm{~nm}$. On the other hand, $G$ increases with decreasing period and reaches $\sim 1.47$ at the period of $20 \mathrm{~nm}$. However, the line width of the metal wires will be only $9 \mathrm{~nm}$ at this short period, which is too small to fabricate by using the present nanoimprint lithography technique. In addition, the issue of aluminum oxidization should be studied for the metal wires at this narrow line width. In spite of these optimization processes, the optical gain $G$ of WGP is not expected to exceed 1.5, smaller than that of DBEF. It was found that the gain factor did not become higher than 1.5 even if both the duty cycle and the period were optimized simultaneously. This is consistent with the result that the internal loss (absorption) of WGP is three times larger than that of DBEF as shown in Table 1, which is the main origin of its lower transmission for the $I_{\mathrm{H}}$ polarization.

\section{CONCLUSION}

The optical performances of WGP were investigated for backlight applications as a reflective polarizer by experiment and the time-domain simulation method. The results were compared with those of DBEF, the commercial dielectric multilayer film. The contrast ratio of WGP was higher than that of DBEF, while its luminance gain factor was 
lower than DBEF by $18 \%$. The light loss factors of DBEF and WGP were approximately 0.03 and 0.10 , respectively, which were obtained by combining the experimental results and FDTD-based simulation. This was attributed to the higher internal loss in metal wires of WGP because of the light absorption via dissipation processes. The period and the width of metal wires comprising WGP were optimized by the FDTD simulation, and the luminance gain factor was found to increase to about 1.47 at the condition of period of $20 \mathrm{~nm}$ at the duty cycle of 0.45 and the height of $155 \mathrm{~nm}$.

\section{REFERENCES}

1. B. H. Hong, "The structural principle and the technological trend of LCD backlights," Journal of the KIIEE 21, 21-36 (2007).

2. G. Park, T. S. Aum, J. H. Kwon, J. H. Park, B. K. Kim, and J. K. Shin, "Characterization and modeling light scattering in diffuser sheets," J. Korean Phys. Soc. 54, 44-48 (2009).

3. J.-H. Ko, J. S. Ryu, M.-Y. Yu, S.-M. Park, and S. J. Kim, "Initial photometric and spectroscopic characteristics of 55inch CCFL and LED backlights for LCD-TV applications," Journal of the KIIEE 24, 8-13 (2010).

4. J.-H. Park, J. H. Lee, J. H. Jeong, K.-B. Nahm, and J.-H. Ko, "Optical simulation study on the performances of collimating films for LCD backlight applications," Hankook Kwanghak Hoeji (Korean J. Opt. Photon.) 18, 432-440 (2007).

5. P. Watson and G. T. Boyd, Mobile Displays, A. K. Bhowmik, et al. ed. (John Wiley \& Sons, Atrium, USA, 2008), Chapter
7, p. 211.

6. M. F. Weber, C. A. Stover, L. R. Gilbert, T. J. Nevitt, and A. J. Ouderkirt, "Giant birefringent optics in multilayer polymer mirrors," Science 287, 2451-2456 (2000).

7. I. Amimori, N. V. Priezjev, R. A. Pelcovits, and G. P. Crawford, "Optomechanical properties of stretched polymer dispersed liquid crystal films for scattering polarizer applications," J. Appl. Phys. 93, 3248-3252 (2003).

8. S. H. Kim, J.-D. Park, and K.-D. Lee, "Fabrication of a nano-wire grid polarizer for brightness enhancement in liquid crystal display," Nanotechnology 17, 4436-4438 (2006).

9. J. R. Park, G. Ryu, J. Byun, H. Hwang, S. T. Kim, and I. Kim, "Numerical modeling and simulation of a cholesteric liquid crystal polarizer," Opt. Rev. 9, 207-212 (2002).

10. M.-Y. Yu, J.-H. Park, and J.-H. Ko, "Study on the luminance properties of optical films for flat-lamp backlight applications,” J. Opt. Soc. Korea 12, 174-177 (2008).

11. B.-W. Lee, M.-Y. Yu, and J.-H. Ko, "Dependence of the gain factor of the reflective polarizer on the configuration of optical sheets," J. Inform. Display 10, 28-32 (2009).

12. M.-Y. Yu, B.-W. Lee, J. H. Lee, and J.-H. Ko, "Correlation between the optical performances of the reflective polarizer and the structure of LCD backlight," J. Opt. Soc. Korea 13, 256-260 (2009).

13. J. S. Ryu, M.-Y. Yu, S.-M. Park, S. J. Kim, and J.-H. Ko, "Study on the correlation between the optical performance of the reflective polarizer and the structure of the backlight for large-size LCD applications," New Physics: Sae Mulli 60, 56-62 (2010).

14. J. J. Wang, L. Chen, X. Liu, P. Sciortino, F. Liu, F. Walters, and X. Deng, "30-nm-wide aluminum nanowire grid for ultrahigh contrast and transmittance polarizers made by UV-nanoimprint lithography," Appl. Phys. Lett. 89, 141105 (2006). 